\title{
Impurity level lifetime measurements using a lock-in amplifier
}

Kenneth Edmondson, Steve Agoston, and Radha Ranganathan

Citation: American Journal of Physics 64, 787 (1996);

View online: https://doi.org/10.1119/1.18176

View Table of Contents: http://aapt.scitation.org/toc/ajp/64/6

Published by the American Association of Physics Teachers

\section{Articles you may be interested in}

Improving student understanding of lock-in amplifiers

American Journal of Physics 84, 52 (2015); 10.1119/1.4934957

An introduction to phase-sensitive amplifiers: An inexpensive student instrument

American Journal of Physics 43, 801 (1998); 10.1119/1.9690

Frequency-domain description of a lock-in amplifier

American Journal of Physics 62, 129 (1998); 10.1119/1.17629

A basic lock-in amplifier experiment for the undergraduate laboratory

American Journal of Physics 71, 1208 (2003); 10.1119/1.1579497

Lock-in detection with DataStudio

American Journal of Physics 74, 207 (2006); 10.1119/1.2167763

Learning the Art of Electronics: A Hands-on Lab Course

American Journal of Physics 85, 78 (2016); 10.1119/1.4966629

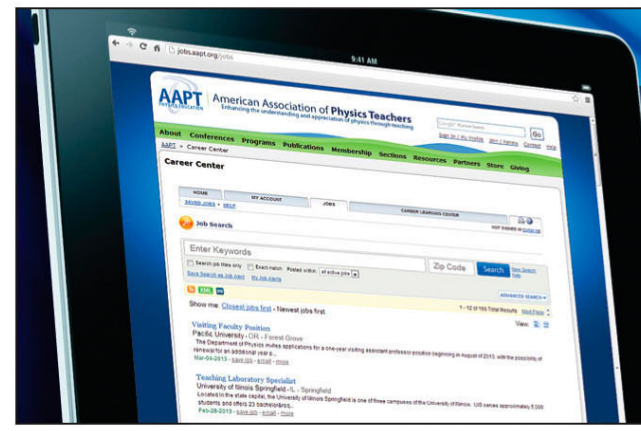

American Association of Physics Teachers

Explore the AAPT Career Center access hundreds of physics education and other STEM teaching jobs at two-year and four-year colleges and universities. 
perbolic relationship perplexing to advocates of Newton's corpuscular model of light. Consider, for example, an infinitely long parallel-edged slit of width $d$. Let $r_{1}$ and $r_{2}$ be respectively the distances of the top edge and bottom edge of the slit from an image point $P$ defined by coordinates $y$ (displacement from the incident forward direction of the light beam) and $z$ (distance of viewing screen from the aperture). Then for fixed $\lambda$ and integer $n$ the relation $r_{2}-r_{1}=n \lambda$, which defines a hyperbolic locus of points
$P$ in the $y z$ plane, locates a specific fringe minimum. To a good approximation the points of maximum light intensity also trace out a hyperbolic trajectory as $z$ is varied. If, however, light were a stream of particles acted upon by short-range forces, then one would expect the particles, after deviation by the knife edges, to propagate through space in a straight line. To account for a hyperbolic trajectory would require some kind of retarding medium (aether) in lieu of empty space.

\title{
Impurity level lifetime measurements using a lock-in amplifier
}

\author{
Kenneth Edmondson, Steve Agoston, and Radha Ranganathan \\ Department of Physics and Astronomy, California State University Northridge, Northridge, \\ California 91330-8268
}

\begin{abstract}
(Received 7 July 1995; accepted 20 October 1995)
Lifetimes of metastable levels of chromium in ruby are determined using an unusual technique. The fluorescence from the chromium is measured using an ac technique in which the excitation is modulated and the fluorescence is detected synchronously. The novelty lies in the measurement of the dependence of the signal on the modulation frequency and extracting the lifetime from the frequency dependence. The experiments and data analysis demand a detailed understanding of the methods of lock-in amplification and synchronous detection and hence they can serve as a useful primer on the subject. The observed values for the lifetimes show excellent agreement with previous measurements. (C) 1996 American Association of Physics Teachers.
\end{abstract}

\section{INTRODUCTION}

This paper describes a novel approach to the measurement of the lifetimes of excited states of impurities in optical materials. The fluorescence emitted by the impurities is measured using ac detection with a lock-in amplifier (LIA). ${ }^{1}$ The material studied in this work is ruby containing $0.05 \mathrm{wt}$. \% of chromium. These experiments not only yield interesting data, but they also demonstrate the essential function of an LIA, since the extraction of the lifetime from the measured signals requires a detailed analysis of the basic operation of an LIA. This method differs from the usual techniques for measuring lifetimes. The experiments and analysis thus serve as an exposition of the methods of phase sensitive detection (PSD). ${ }^{1}$ This paper is organized in four sections. We begin with a brief review of the energy level structure of chromium in ruby, followed by a discussion of the operation of an LIA. In Sec. III we develop a model that relates the measured quantities to the lifetime. A description of the experimental set up and results for the lifetime are presented in Sec. IV. Section $V$ concludes with a critique of the method and simplifications for its adaptability to other laboratory environments.

\section{SAMPLE DESCRIPTION AND EXPERIMENTAL TECHNIQUE}

Ruby is crystalline $\mathrm{Al}_{2} \mathrm{O}_{3}$ (sapphire) in which some of the aluminum atoms are replaced by chromium atoms, which are responsible for the color of ruby. Ruby is a solid-state laser material. ${ }^{2}$ The energy level scheme of $\mathrm{Cr}^{3+}$ in ruby is shown in Fig. $1 .^{3}$ At room temperature most of the ions are in the ground state, denoted by $g$. The excited states form bands of levels. $\mathrm{A} \mathrm{Cr}^{3+}$ ion can be excited into the "green band" or the "blue band" by optically pumping with light of wavelength 400 to $700 \mathrm{~nm}$. The excited ions decay rapidly into one of the metastable $R$ levels. From the $R$ levels the ions decay radiatively back into the ground level, ${ }^{4}$ emitting two fluorescence lines in the wavelength region of 692 to 695 $\mathrm{nm}$. Ruby serves as the classic example of a three-level laser. ${ }^{2}$ The lasing occurs between an $R$ level and the ground level. The former are characterized by long lifetimes $(\sim 3.79$ $\pm 0.14 \mathrm{~ms}$ at ambient pressure and room temperature). ${ }^{5-7}$ It is this long upper-level lifetime that makes ruby an ideal laser material. The interaction that leads to the fluorescence emission (or atomic transition) is electric dipole in nature, and the radiative lifetime is inversely proportional to the transition probability between the upper and the lower levels. $^{8}$

Normally lifetimes are obtained by time-resolved spectroscopy, in which the atoms are pumped by a pulsed laser and the time decay of the resulting fluorescence signal is measured. In this case, the width of the pump pulse must be much shorter than the lifetime as indicated in Fig. 2. In the case of ruby the time dependence of the decaying fluorescence is exponential, ${ }^{5,6}$

$$
I=I_{0} \exp \left(\frac{-t}{\tau}\right)
$$

where $I$ is the fluorescence intensity after a time $t$ following the excitation. The decay time or lifetime $\tau$ is obtained by line fitting. The fluorescence spectrum, on the other hand, is 


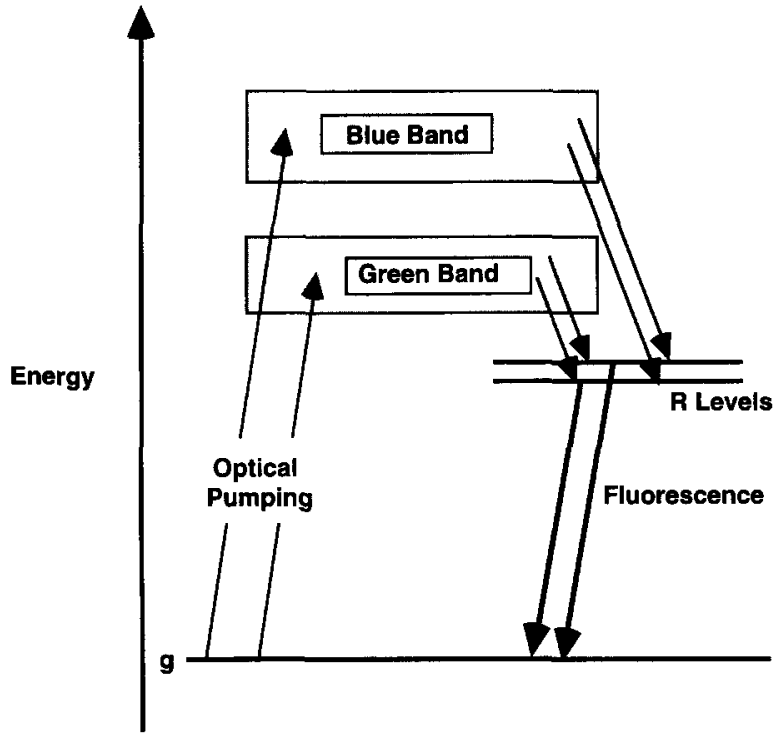

Fig. 1. Energy levels of $\mathrm{Cr}^{3+}$ in ruby.

usually measured by monitoring steady state fluorescence, in which the pumping is performed by a CW source; the resulting emission spectrum yields transition energies rather than the lifetimes. In measuring CW signals, ac detection using an LIA can be employed to enhance the signal-to-noise ratio. In this method, a mechanical chopper is used to turn the excitation beam on and off. Thus the sample is pumped in a periodic fashion, and the excitation can be viewed as a square wave. The emitted fluorescence, also periodic, is then dispersed by a spectrometer and monitored by a detector whose response time is shorter than the time period of the signal. The detector's output serves as input signal to the LIA. An auxiliary output from the chopper, a square wave called the reference signal, provides the reference input to the LIA. The basic requirement of an LIA is that the input signal be periodic ("ac") and synchronous with the reference signal; hence the term "ac detection." The purpose of the LIA is the enhancement of the signal-to-noise ratio. In an LIA the signal component of the input is enhanced relative to any noise by PSD, and a dc output proportional to the am-

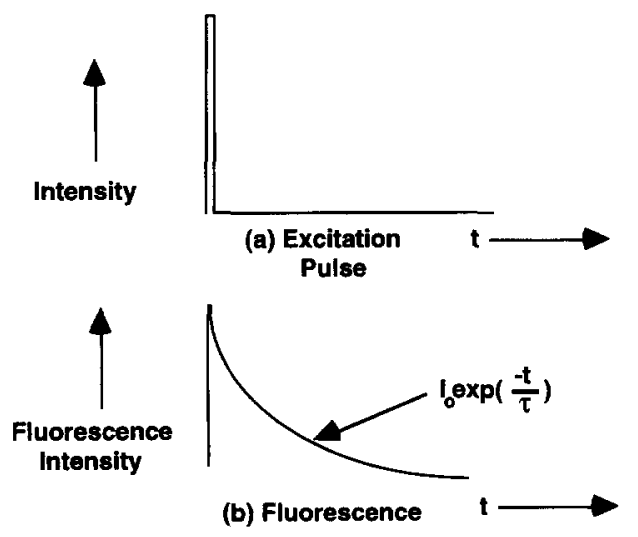

Fig. 2. Determination of lifetime using a pulsed laser and time resolved measurements. (a) A narrow excitation pulse and (b) the time decay of the resulting fluorescence.

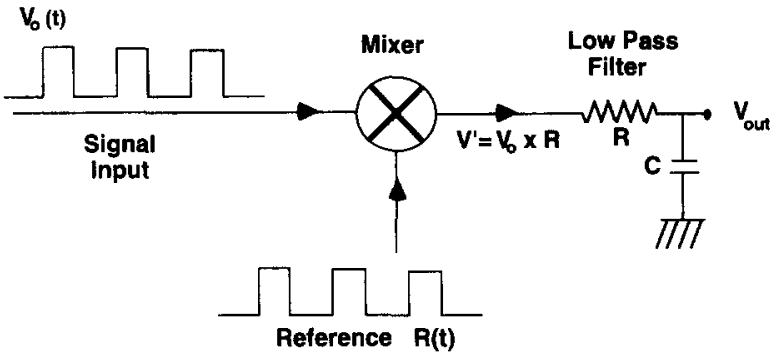

Fig. 3. Elements of phase sensitive detection.

plitude of the input signal is obtained. The essential elements of PSD, performed by an LIA, are shown in Fig. 3. The mixer receives the two synchronous inputs-reference $R$ and signal $V_{0}$. In Fig. 3, $V_{0}$ is shown to be a square wave, but it need not be. These two signals are multiplied by the mixer and an output $V^{\prime}=V_{0} \times R$ is produced. Since both $R(t)$ and $V_{0}(t)$ are periodic with the same fundamental frequency, they can be decomposed into Fourier components. Assuming the reference signal $R(t)$ to be a square wave of unit height, its expansion contains only the odd harmonics,

$$
R(t)=\frac{1}{2}+\frac{2}{\pi} \sum_{n=0}^{\infty} \frac{1}{2 n+1} \sin (2 n+1)(2 \pi f t+\phi),
$$

where $f$ is the fundamental (chopping) frequency, and $\phi$ is the phase difference between $R(t)$ and $V_{0}(t)$. The Fourier series for $V_{0}$ assumes the form,

$$
V_{0}(t)=\frac{a_{0}}{2}+\sum_{m=1}^{\infty}\left(a_{m} \cos (2 m \pi f t)+b_{m} \sin (2 m \pi f t)\right)
$$

To determine the coefficients $a_{m}$ and $b_{m}$, one needs a model for the signal $V_{0}(t)$ for the time interval $0 \leqslant t \leqslant T$ (where $T=1 / f)$. The output of the mixer, $V^{\prime}$, being a product of the two sums in Eqs. (2) and (3), will contain terms that are products of two harmonic terms. A typical term is

$$
\begin{aligned}
& \frac{1}{2 n+1} \sin ((2 n+1)(2 \pi f t+\phi)) a_{m} \cos (2 m \pi f t) \\
& =\frac{a_{m}}{2(2 n+1)}\left\{\begin{array}{c}
\sin ((2 n+1)(2 \pi f t+\phi)+2 m \pi f t)+ \\
\sin ((2 n+1)(2 \pi f t+\phi)-2 m \pi f t)
\end{array}\right\},
\end{aligned}
$$

where the identity $\sin A \cos B=(1 / 2) \quad\{\sin (A+B)+\sin (A$ $-B)\}$ has been used to obtain the right-hand side. This expression shows that $V^{\prime}(t)$ contains harmonic terms whose frequencies are the sums and differences of the frequencies of the individual harmonic terms in $V_{0}(t)$ and $R(t)$. The crucial point is that whenever $(2 n+1)=m$, the difference terms $[\sin (A-B)$ or $\cos (A-B)]$ will become zero frequency or dc terms. Hence when $V^{\prime}$ passes through a low-pass filter (the next stage of PSD), all the high frequency terms (the sum frequencies) are rejected and only the dc terms pass through. The latter have the form $\left(a_{m} \sin \phi+b_{m} \cos \phi\right)$, where $m$ is odd. The coefficients $a_{m}$ and $b_{m}$ in general decrease with $m$ and the output, $V_{\text {out }}$, of the PSD can be approximated by $\left(a_{1} \sin \phi+b_{1} \cos \phi\right)$. The LIA allows for an adjustment of the phase angle $\phi$, so that $V_{\text {out }}$ can be maximized. The measured quantity is thus

$$
V_{\text {out }} \approx\left(a_{1}^{2}+b_{1}^{2}\right)^{1 / 2} \text {. }
$$




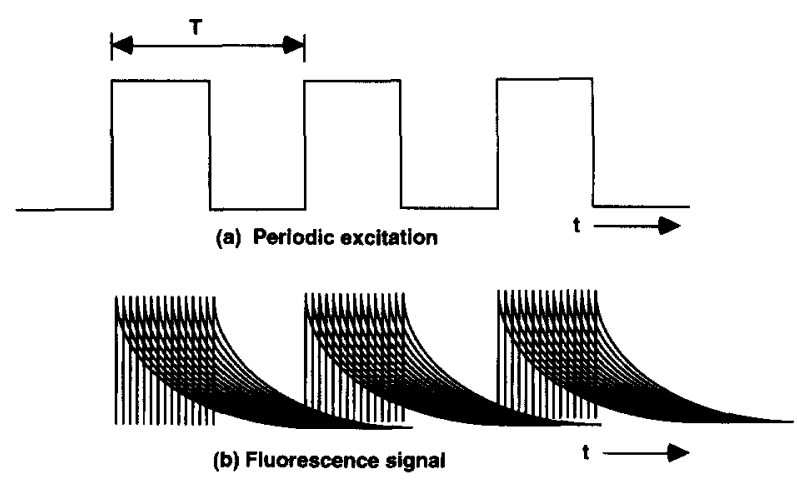

Fig. 4. CW fluorescence in which the excitation is periodic. (a) The periodic excitation produced by a chopper. (b) The periodic fluorescence signal, showing the time dependence of the signal in any one period.

The primary purpose of PSD, however, is noise suppression. All asynchronous noise terms in the input, when multiplied with $R$ in the mixer, yield sum and difference frequencies, none of which is dc and hence these noise terms are filtered by the low-pass filter. We now proceed to obtain the coefficients $a_{m}$ and $b_{m}$ and $V_{\text {out }}$ for those situations where fluorescence decay can be expressed by Eq. (1).

\section{MODEL FOR $V_{0}(t)$}

A laser excitation of the sample for a period of time $T$ is shown in Fig. 4(a). The $\mathrm{Cr}^{3+}$ ions are excited into the green band by the $514.5 \mathrm{~nm}$ green line of an argon laser. The ions rapidly decay into the $R$ levels and then emit the red fluorescence as they relax into the ground state. Since the filling of the $R$ levels is a rapid process, the rise time of the fluorescence signal can be treated as instantaneous. In the first half of a period during which the laser is on, excitation and decay occur continuously, but some of the fluorescence persists into the second half of the cycle. This behavior is depicted in Fig. 4(b). For an excitation at a time $t^{\prime}$, the emitted intensity at a later time $t$ will be

$$
I\left(t, t^{\prime}\right)=I_{0} \exp \frac{-\left(t-t^{\prime}\right)}{\tau}
$$

The intensity at any time $t$ in the interval $0<t<T / 2$ is the integrated sum of intensities at time $t$ due to emissions from ions excited at all previous times, $t^{\prime}<t$. At any time $t$ in the interval $T / 2$ to $T$, the intensity is a similar sum of emissions from all excitations that occurred at $t^{\prime}<T / 2$. These statements are expressed by,

$$
\begin{gathered}
I(t)=\int_{0}^{t} d I\left(t, t^{\prime}\right)=\frac{I_{0}}{\tau} \int_{0}^{t} \exp \left(\frac{-\left(t-t^{\prime}\right)}{\tau}\right) d t^{\prime}, \\
0 \leqslant t \leqslant T / 2,
\end{gathered}
$$

and

$$
\begin{gathered}
I(t)=\int_{0}^{T / 2} d I\left(t, t^{\prime}\right)=\frac{I_{0}}{\tau} \int_{0}^{T / 2} \exp \left(\frac{-\left(t-t^{\prime}\right)}{\tau}\right) d t^{\prime}, \\
T / 2 \leqslant t \leqslant T .
\end{gathered}
$$

Integration yields,

$$
I(t)=I_{0}\left\{1-\exp \frac{-t}{\tau}\right\}, \quad \text { for } 0 \leqslant t \leqslant T / 2,
$$

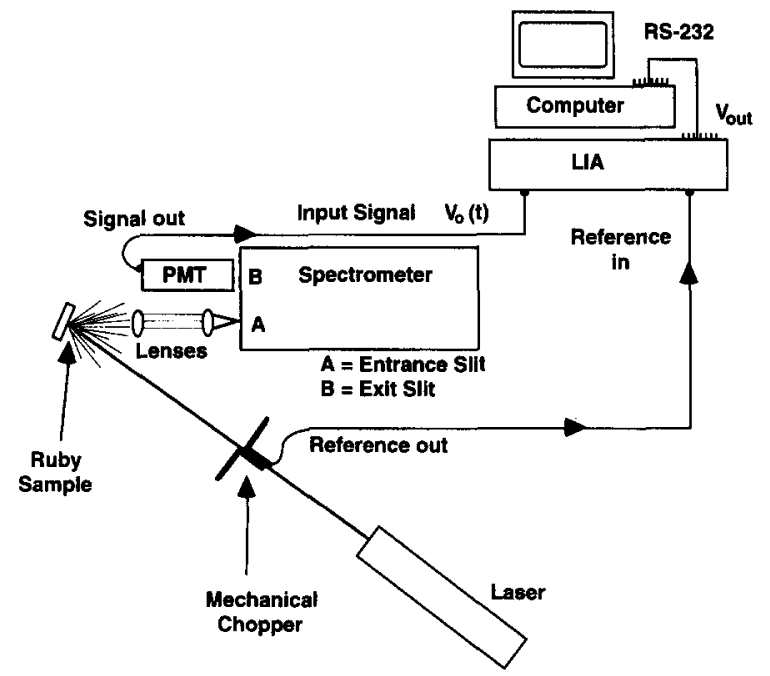

Fig. 5. Experimental set up for $\mathrm{CW}$ fluorescence measurements.

and

$$
I(t)=I_{0}\left\{\left(\exp \frac{T}{2 \tau}-1\right) \exp \frac{-t}{\tau}\right\}, \quad \text { for } T / 2 \leqslant t \leqslant T .
$$

The signal $V_{0}(t)$ is proportional to and a measure of $I(t)$, so that $V_{0}(t)$ and $I(t)$ have identical periodic behavior and the same Fourier coefficients, which are

$$
a_{m}=\frac{2}{T} \int_{0}^{T} I(t) \cos (2 m \pi f t),
$$

and

$$
b_{m}=\frac{2}{T} \int_{0}^{T} I(t) \sin (2 m \pi f t) .
$$

Substitution of Eqs. (7) and (8) into Eqs. (9) and (10) and some straightforward integration yield expressions for $a_{m}$ and $b_{m}$. The leading coefficients $(m=1)$ are

$$
a_{1}=2 I_{0} F(f, \tau) \text {, }
$$

and

$$
b_{1}=2 I_{0}\left[\frac{1}{\pi}+2 \pi f \tau F(f, \tau)\right],
$$

where

$$
F(f, \tau)=f \tau \frac{[\exp (-1 / f \tau)-\exp (-1 / 2 f \tau)-2]}{\left[1+4 \pi^{2} f^{2} \tau^{2}\right]} .
$$

Substitution of these expressions into Eq. (5) gives the measured signal,

$$
V_{\text {out }}=2 I_{0}\left[F^{2}(f, \tau)+\left\{\frac{1}{\pi}+2 \pi f \tau F(f, \tau)\right\}^{2}\right]^{1 / 2},
$$

Clearly $V_{\text {out }}$ depends on the chopping frequency $f$.

We now consider some of the limiting cases. If $\tau \ll T$, then $I(t)=I_{0}$ for $0 \leqslant t \leqslant T / 2$ and $I(t)=0$ for $T / 2 \leqslant t \leqslant T$. That is, $I(t)$ or $V_{0}(t)$ is a square wave just like $R$. Furthermore $a_{1}=0, b_{1}=2 I_{0} / \pi, V_{\text {out }} \sim I_{0}$, and the signal is independent of frequency. This is the situation normally encountered in $\mathrm{CW}$ fluorescence measurements. However in the case of ruby where $\tau \approx 3.8 \mathrm{~ms}, V_{\text {out }}(t)$ will depend on $f$, even for low 


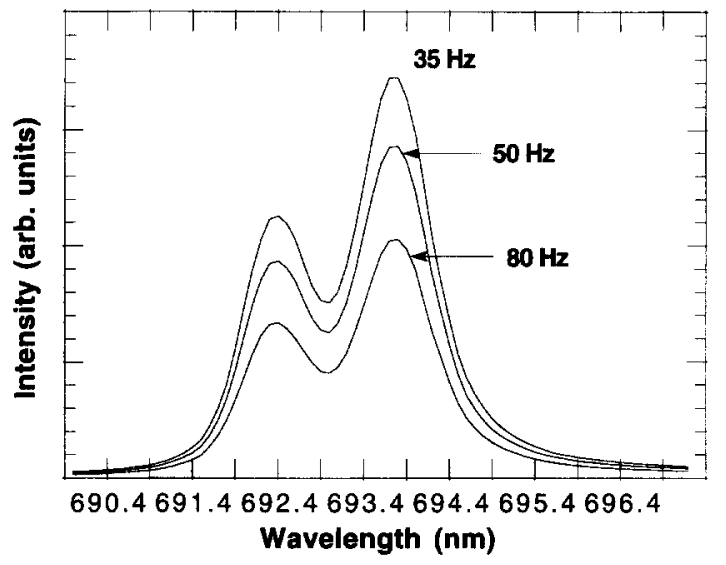

Fig. 6. Room temperature ruby fluorescence spectra for three chopping frequencies.

values of $f$. By studying the dependence of $V_{\text {out }}(t)$ on $f$ and fitting the observed behavior using Eq. (14), the lifetime $\tau$ can be obtained. We thus have a method to determine $\tau$ using CW spectroscopy. The results of our measurements are given in the following section.

\section{EXPERIMENTAL RESULTS AND DISCUSSION}

The experimental arrangement is shown in Fig. 5. The chromium ions in the sample are pumped from the ground level to the green band by the $514.5 \mathrm{~nm}$ line of a CW argon laser. The excitation power density was $75 \mathrm{~mW} \mathrm{~cm}^{-2}$. A variable frequency chopper (Ithaco Model $383 \mathrm{~A}$ and B) interrupts the laser beam prior to incidence on the sample. The emitted fluorescence is focused on the entrance slit of a 0.75 m Spex Czerny Turner monochromator and a photomultiplier (PMT) tube at its exit slit detects the radiation passed by the spectrometer. The output of the PMT is connected to the LIA (Model 5209, EG \& G Princeton Applied Research) as input. Capture of the dc signal, $V_{\text {out }}$, from the LIA, is performed by an IBM-compatible 286 computer linked to the LIA via RS-232 ports. Data acquisition software controls the stepper motor that turns the grating in the spectrometer and plots $V_{\text {out }}$ (proportional to the fluorescence intensity) vs. wavelength on the screen. As shown in Fig. 6, room tem-

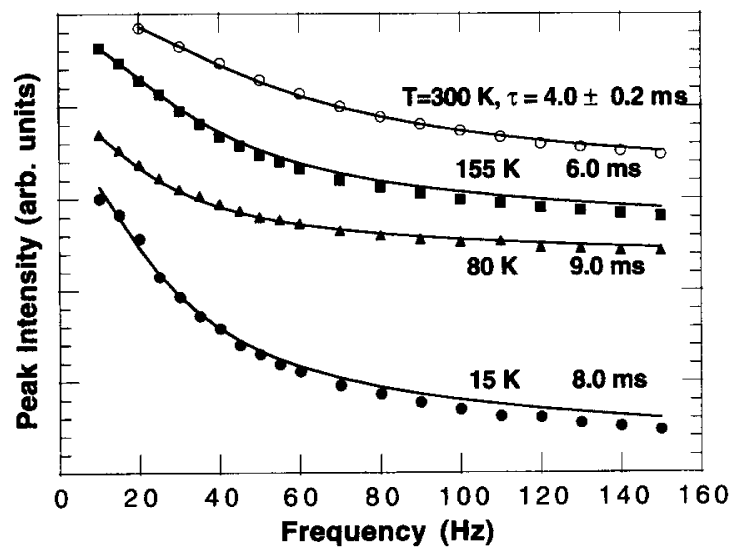

Fig. 7. The symbols are the fluorescence peak intensities of the $6938 \AA$ line as a function of the chopping frequency. The solid line is a fit to Eq. (15).

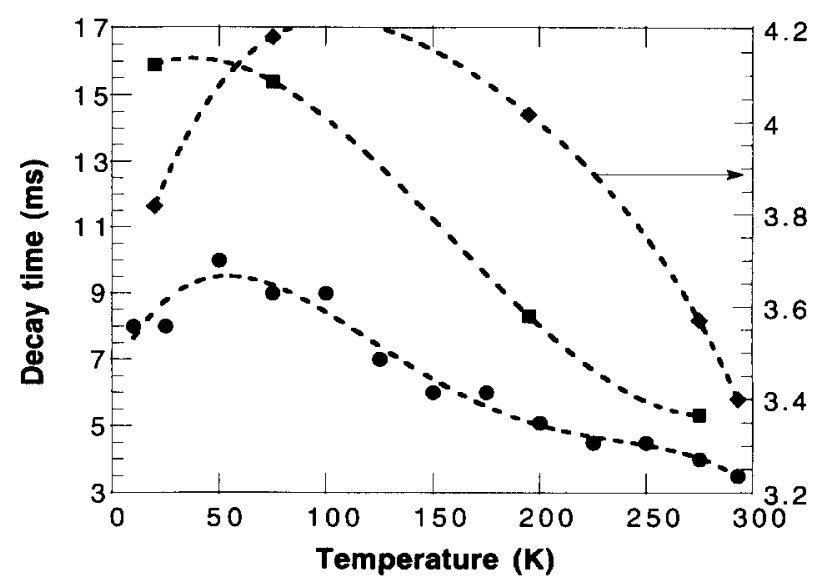

Fig. 8. Comparison of the temperature dependence of $\tau$ obtained in this work () with that of Ref. 5, for a large rod sample ( $\mathbf{0}$ ) and for a small dilute sample ( , refer to right side axis). The dashed lines are meant only to guide the eye. The values determined in this work are shorter than those for the large rod and longer than those for the small, dilute sample. The differences are due to effects of self absorption which increase with size and $\mathrm{Cr}^{3+}$ concentration.

perature fluorescence spectra, obtained for three different chopping frequencies, exhibit the familiar ruby peaks at 692.4 and $693.8 \mathrm{~nm}$. Note that the fluorescence decreases with frequency in agreement with the behavior predicted by our analysis in the previous section. Chopping frequencies range from 10 to $200 \mathrm{~Hz}$. The peak height vs. the frequency for the $693.8 \mathrm{~nm}$ line is plotted in Fig. 7 (shown by symbols). Equation (14) is fit to this lineshape, and the best fit is shown by the solid line. The fitting process returns a value of $\tau=4.0$ $\pm 0.2 \mathrm{~ms}$ at room temperature. This is in excellent agreement with the recently reported value of $3.79 \pm 0.14 \mathrm{~ms}{ }^{7}{ }^{7}$ Measurements were also made at temperatures down to $10 \mathrm{~K}$ for comparison with the existing data. The frequeny dependence of the peak intensities and the corresponding fits for the lower temperatures are shown in Fig. 7. The values of $\tau$ returned by the fits at each temperature are compared with the existing values of the decay time in Fig. 8 (Ref. 5). The following observations are noteworthy: Strictly speaking, the quantity $\tau$ determined experimentally is the fluorescence decay time rather than the lifetime. In the case of ruby, selfabsorption of the fluorescence and re-emission can lengthen the decay time. ${ }^{5}$ This is particularly important for $T \leqslant 150 \mathrm{~K}$. Furthermore, the decay time depends on the chromium concentration and the size of the sample, and it increases with the size, varying at $77 \mathrm{~K}$ from $4.3 \pm 0.3 \mathrm{~ms}$ in a dilute dispersed powder to $15 \mathrm{~ms}$ in a boule having about $1.6 \pm 10^{19}$ $\mathrm{cm}^{-3}$ of chromium. ${ }^{9}$ The sample in this work is a $4 \times 4 \times 2$ $\mathrm{mm}$ faceted crystal. The comparison in Fig. 8 is with the decay time measured by Nelson and Sturge for a $1.59 \mathrm{~cm}$ diameter ruby rod of length $18.9 \mathrm{~cm}$, containing $1.8 \times 10^{19}$ $\mathrm{cm}^{-3} \mathrm{Cr}^{3+}$. The shorter decay times reported here are attributed to the smaller size of our crystal. Nelson and Sturge have measured the lifetime for optically thin, small, dilute crystals (under conditions for which self-absorption is negligible) and find that the lifetime lengthens as the temperature is lowered, from a room temperature value of about $3.4 \mathrm{~ms}$, passing through a maximum of about $4.2 \mathrm{~ms}$ below $100 \mathrm{~K}$ and shortens again at lower temperatures. The decay times we find are longer than that in the small, dilute samples and are attributed to self absorption. In many fluorescent solids 
the lifetime decreases monotonically as the temperature is lowered, while in ruby it passes through a maximum. Such a temperature dependence was observed by Nelson and Sturge. We also observe a maximum in $\tau$ at about $50 \mathrm{~K}$. As regards the fits in Fig. 7, we note that the fitted lines deviate from the data points at the higher frequencies. This is because the model assumes that the fluorescence decays sufficiently within a period, that is $T / 2>\tau$ [or $f<1 /(2 \tau)]$, so that the signal from that period is negligible in the next period.

\section{CRITIQUE AND CONCLUSIONS}

The results of these experiments demonstrate the applicability of this new technique. Equation (14), however, applies only where the fluorescence decay is exponential. In other cases, the Fourier coefficients must be determined in the individual situations and used in Eq. (5). Since an LIA normally functions only up to a hundred $\mathrm{kHz}$, the $\tau$ to be measured must be greater than $10 \mu \mathrm{s}$. Commercial instruments that use different technology are available which can extend the measurement frequency range to $\mathrm{MHz}$ or even $\mathrm{GHz}$ frequencies. This will enable the observation of more rapid phenomena, in which case electro-optic modulation rather than mechanical chopping will be needed.

We now suggest some simplifications that will make the experiments more accessible. Instead of pumping with an argon laser, the fluorescence can be excited by a $5 \mathrm{~mW}$ He-Ne laser or focused white light, which are more commonly available in a student laboratory. Measurement of the full spectrum is not necessary. If a spectrometer is available, then the grating may be positioned for obtaining the peak intensity at the peak wavelength ( $6938 \AA$ ), because only the peak intensity is relevant and $V_{\text {out }}$ can be read from the output display of the LIA. This eliminates the need for scanning and the complexities of data acquisition. In the absence of a spectrometer, an appropriate band pass filter may be used. The experiments are feasible even if neither a spectrometer nor a band pass filter is available. In such a case, the emitted fluorescence can be directly focused on the detector. However a filter that cuts off the scattered incident light is needed. It is essential that all scattered light be eliminated for accurate lifetime determination. The low temperature mea- surements were performed only for the purpose of having more data to illustrate the method. The PMT used in our experiments has a fast response time of $2 \mathrm{~ns}$, and the flatness of its frequency response for the frequency range of the experiments was verified. An ordinary silicon photo-detector could be used as well, but its frequency response must be determined and included as a factor in Eq. (14).

The experiments described here are meant to provide insight into the function of an LIA, which is commonly used in areas where one is detecting weak signals. We have shown how an understanding of the basics of lock-in amplification can lead to the measurements of physical quantities that are not normally determined by phase sensitive detection.

\section{ACKNOWLEDGMENTS}

Reassigned time for this work was provided by the Office of Research and Sponsored Projects, the Affirmative Action Faculty Development Program and the School of Science and Mathematics.

\footnotetext{
${ }^{1} \mathrm{P}$. Horowitz and W. Hill, The Art of Electronics (Cambridge U.P., Cambridge, 1980), pp. 628-631.

${ }^{2}$ P. W. Milonni and J. H. Eberly, Lasers (Wiley, New York, 1988), pp. 308-310.

${ }^{3}$ R. M. Macfarlane, "Analysis of the Spectrum of $d^{3}$ Ions in Trigonal Crystal Fields," J. Chem. Phys. 39, 3118-3126 (1963).

${ }^{4}$ T. H. Maiman, R. H. Hoskins, I. J. d'Haenens, C. K. Asawa, and V. Evtuhov, "Stimulated Optical Emission in Fluorescent Solids. II. Spectroscopy and Stimulated Emission in Ruby,' Phys. Rev. 123, 1151-1157 (1961).

${ }^{5}$ D. F. Nelson and M. D. Sturge, "Relation between Absorption and Emission in the Region of the $R$ Lines of Ruby," Phys. Rev. 137A, 1117-1130 (1965).

${ }^{6}$ J. H. Eggert, K. A. Goettel, and I. F. Silvera, "Ruby at high pressure. II. Fluorescence lifetime of the $R$ line to $130 \mathrm{GPa}$," Phys. Rev. B 40, 57335738 (1989).

${ }^{7}$ A. Mandelis, Z. H. Chen, and R. Bleiss, "Quantum efficiency and metastable lifetime measurements in ruby $\left(\mathrm{Cr}^{3+}: \mathrm{Al}_{2} \mathrm{O}_{3}\right)$ via lock-in ratewindow photothermal radiometry," J. Phys. 4, 401-404 (1994).

${ }^{8} \mathrm{~S}$. Sugano, Y. Tanabe, and H. Kamimura, Multiplets of Transition-Metal lons in Crystals (Academic, New York, 1970), pp. 113-118.

${ }^{9}$ F. Varasanyi, D. L. Wood, and A. L. Schawlow, "Self-absorption and trapping of sharp-line resonance radiation in ruby," Phys. Rev. Lett. 3, 544-545 (1959).
}

\section{SYMMETRY LAWS}

The existence of symmetry laws is in full accordance with our daily experience. The simplest of these symmetries, the isotropy and homogeneity of space, are concepts that date back to the early history of human thought. The invariance of physical laws under a coordinate transformation of uniform velocity, also known as the invariance under Galilean transformations, is a more sophisticated symmetry that was early recognized, and formed one of the corner-stones of Newtonian mechanics. Consequences of these symmetry principles were greatly exploited by physicists of the past centuries and gave rise to many important results. A good example in this direction is the theorem that in an isotropic solid there are only two elastic constants.

Chen Ning Yang, "The law of parity conservation and other symmetry laws of physics"' (Nobel Lecture, December 11, 1957, reprinted in Nobel Lectures, Physics, Vol. 3, 1942-1962, Elsevier Amsterdam, 1964). 